\title{
Le Visiteur d'Eric-Emmanuel Schmitt Lecture psychocritique
}

\section{* Dr. Rana HARB}

\section{Résumé}

Romancier, dramaturge, conteur, en deux décennies, Eric-Emmanuel Schmitt est devenu un des auteurs francophones les plus lus et les plus représentés dans le monde. Le Visiteur, sujet de la présente étude, présente Sigmund Freud, enfermé dans son salon avec un visiteur, un inconnu dont l'identité s'avère mystérieuse.

Nous tâcherons en premier lieu, en nous basant sur la psychocritique de Charles Mauron, d'élucider les différentes identités qu'acquiert l'inconnu. Nous analyserons ensuite les différentes techniques de cures psychanalytiques appliquées par Freud sur l'Inconnu. Nous tenterons ensuite de détecter les réseaux d'associations à travers les groupements d'images obsédantes et involontaires qui se dégagent du texte. Nous tracerons ensuite le mythe personnel de l'auteur à travers les situations et les modifications de structures dans la pièce. Schmitt conjecture alors un cycle qu'il appelle le « Cycle de l'Invisible » qui est la recherche du sens de l'existence, une existence qui doit être uniquement détectée par l'esprit. Schmitt souligne l'importance de la sagesse spirituelle et son rôle dans la vie de l'homme. La dernière partie de ce travail sera orienté vers l'étude du Cycle de l'Invisible dans Le Visiteur d'Eric-Emmanuel Schmitt.

Schmitt ne cherche aucunement à convaincre son lecteur de sa foi, mais il demande à son lecteur de partager sa pensée. C'est dans cette optique que nous délimiterons l'étude du Cycle de l'Invisible d'Eric-Emmanuel Schmitt, tout en proposant quelques pistes de réflexions.

$\mathrm{Au}$ terme de cette étude sur la conception de Schmitt du Cycle de l'Invisible, nous croyons pouvoir conclure que Le Visiteur ne propose aucune réponse définitive. Schmitt invite son spectateur à adopter comme lui une attitude interrogative. Il lui ouvre grandes les portes de la réflexion.

Schmitt puise son optimisme du fond même du malheur. L'espérance est le seul moyen d'apaiser la douleur. L'espérance, c'est croire en cette force majeure qui gère le monde. L'espérance réside dans cette certitude que Dieu est toujours là. C'est surtout, cette touche d'optimisme et cette quête du Bien au milieu du Mal, qui ont permis à Eric-Emmanuel Schmitt, de séduire des milliers de lecteurs à travers le monde.

Avec un style, à la fois, précis et étincelant d'originalité, Schmitt, en allant au cœur du Moi, parvient à briser les barrières imposées par les religions, les dogmes et les différences. Rien ne saurait ébrécher son amour pour l'Autre. La force de son récit ne s'inscrit-elle pas au cœur de sa quête de l'humain universel ? $\mathrm{Ne}$

\section{Maître de conférences- Faculté de Pédagogie- Université d'Alexandrie}


prépare-t-il pas ainsi son lecteur à son nouveau Cycle celui de l'Humain et de l'Inhumain ?

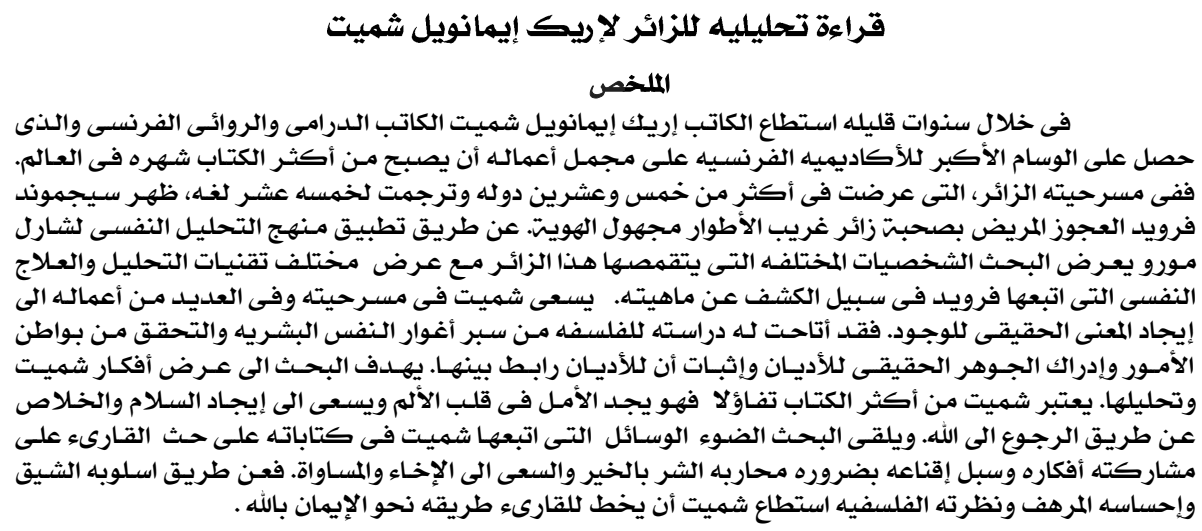

\section{Introduction :}

Entreprendre une étude sur les œuvres d'Eric-Emmanuel Schmitt suscite plusieurs interrogations. Romancier, dramaturge, conteur, en deux décennies, Eric-Emmanuel Schmitt est devenu un des auteurs francophones les plus lus et les plus représentés dans le monde. Ses livres sont traduits en 43 langues et atteignent des tirages exceptionnels. Ses pièces ont été récompensées par plusieurs Molière et le Grand Prix du théâtre à l'Académie française. Faisant revivre des personnages connus à travers les siècles: Don Juan, Diderot, Freud, le lecteur se trouve dans une atmosphère qui lui est familière et étrange à la fois. Son œuvre diversifiée: romans, pièces de théâtre, contes, essais, laisse le lecteur perplexe, assailli par des interrogations philosophiques, psychologiques, voire même métaphysiques. Schmitt n'impose aucune réponse. Cette «énigmaticité » ${ }^{1}$ transporte le lecteur hors du commun et l'incite à partager avec l'auteur ce monde à la fois inquiétant et réconfortant. Pourtant Schmitt ne cherche point à troubler son lecteur. Au contraire, il va jusqu'à la fin 'l'emmener sans lui

\footnotetext{
${ }^{1}$ La notion d'énigmaticité se distingue de la simple énigme. L'énigme use de la devinette et implique sa propre solution. L'énigmaticité est une énigme sans solution, autant dire une énigme qui n'est plus une énigme, mais seulement une question.

Cf. BESSIÈRE, Jean «L’Énigmaticité de Henry James, en passant par la réception française de son œuvre », E-rea [En ligne], 3.2|2005, document 7, mis en ligne le 15 octobre 2005, consulté le 05 avril 2017. URL : http://erea.revues.org/554 ; DOI : 10.4000/erea.554
} 
lâcher la main. Il faut aller jusqu'au bout de l'accompagnement, le conduire à une vision de la condition humaine". ${ }^{2}$

Tout en se basant sur Le Visiteur et La Nuit de feu, la présente étude aura pour objet d'en extraire les éléments essentiels de l'inconscient de l'auteur et tenter de lier les associations secrètes de sa pensée afin de parvenir à démystifier sa création littéraire.

Dans notre démarche nous tenterons de questionner davantage l'œuvre de Schmitt. En fait, la création artistique est pour l'auteur le lieu de la satisfaction de phénomènes inconscients. Nous suivrons l'approche de Charles Mauron pour analyser les textes de Schmitt. Basé sur les théories freudiennes, Mauron affirme que la création littéraire est pour l'auteur le lieu de l'examen de l'inconscience. Les textes de Schmitt ne sont-ils pas une élucidation de son inconscient? $\mathrm{Ne}$ peuvent-ils pas être considérés comme étant une autoanalyse effectuée par l'auteur lui-même ? Ainsi la psychocritique de Charles Mauron s'avère la plus adéquate pour mener cette réflexion. Cette méthode fournira le support théorique nécessaire à la démarche et permettra de décrypter les signes de l'inconscient et déchiffrer des phénomènes qui semblent éloignés les uns des autres mais qui ne sont réellement que le reflet du discours inconscient de l'auteur.

Nous commencerons par une analyse de la pièce de théâtre, Le Visiteur, qui fut présentée en Septembre 1993 au Petit Théâtre de Paris. La pièce est jouée dans une vingtaine de pays et traduite en quinze langues en l'espace de dix ans.

Dans Le Visiteur, Sigmund Freud, vieux malade, persécuté par la Gestapo qui arrête sa fille, lors de l'invasion de l'Autriche par les troupes hitlériennes, se trouve enfermé dans son salon avec un visiteur, un inconnu dont l'identité s'avère mystérieuse. Est-ce un malade, un fou, un visionnaire?

Nous tâcherons en premier lieu d'élucider les différentes identités qu'acquiert l'inconnu tout au long de la pièce, en soulignant l'aspect onirique que Freud lui attribue. Le Visiteur est une pièce énigmatique, située à mi-chemin entre le réel et le rêve, qui puise sa force de la présence du personnage de Freud, père de la psychanalyse ainsi que de la présence de l'Inconnu qui ajoute une dimension

\footnotetext{
${ }^{2}$ VILAS, Jean, «La malédiction du succès » in Les cahiers de la maison, n.103, Novembre 2007 
mystique à la pièce. A ce titre, le spectateur est en droit de se demander s'il ne s'agit pas tout simplement d'un rêve, d'un cauchemar, d'une hallucination...

Nous analyserons ensuite les différentes techniques de cures psychanalytiques adoptées et appliquées par Freud sur l'Inconnu afin de parvenir à dévoiler son secret. La tâche est loin d'être facile car l'Inconnu semble être une incarnation, ou, dans une optique plus sérieuse, Freud se trouve t-il en présence de Dieu ? Rien n'est affirmé.

Schmitt est un auteur énigmatique et mystique, et, à travers l'analyse du personnage de Freud, il serait possible de découvrir le secret de l'angoisse que Schmitt éprouve face à l'existence. A travers cette étude, nous tâcherons d'élucider l'importance de la méthode psychocritique de Charles Mauron dans l'analyse de la pièce. Nous tenterons ensuite de détecter les réseaux d'associations à travers les groupements d'images obsédantes et involontaires qui se dégagent du texte. Nous tracerons ensuite le mythe personnel de l'auteur à travers les situations et les modifications de structures dans la pièce. Nous pourrons ensuite ressortir le discours inconscient qui se trame derrière le discours conscient de l'auteur.

En se basant sur La Nuit de feu, nous rechercherons finalement la correspondance de la situation interne avec la vie de l'auteur. Dans cette œuvre, Schmitt raconte sa randonnée dans le grand désert algérien où il s'égare dans l'immensité du Hoggar et passe une nuit glaciale sans eau ni vivres. Cependant, il n'éprouve aucune peur. Il voit par contre s'ébranler toutes ses certitudes. L'auteur dévoile pour la première fois son intimité spirituelle. Il décrit cette force brûlante qui l'envahit, ce feu qu'il nomme Dieu.

Schmitt conjecture alors un cycle qu'il appelle le «Cycle de l'Invisible ${ }^{3}$ qui est la recherche du sens de l'existence, une existence qui doit être uniquement détectée par l'esprit. Schmitt souligne l'importance de la sagesse spirituelle et son rôle dans la vie de l'homme. Il est à noter que malgré la grande diversité des thèmes dans l'œuvre de Schmitt, cette idée-force ${ }^{4}$

\footnotetext{
${ }^{3}$ MICHEL, Meyer, Eric-Emmanuel Schmitt ou les identités bouleversées, Albin Michel, Paris, 2004, p.11.

${ }^{4}$ Ibid. 
anime le fil de sa pensée. ${ }^{5}$ La dernière partie de ce travail sera orientée vers l'étude du Cycle de l'Invisible. Schmitt ne cherche aucunement à convaincre son lecteur de sa foi, mais il lui demande de partager sa pensée. Il n'impose aucune vérité. Son théâtre est ainsi loin d'être dogmatique. Il invite ainsi son spectateur à écouter, à réfléchir, à dialoguer avec les autres. Schmitt affirme :

«Je ne suis pas un écrivain contagieux ${ }^{6}$

C'est dans cette optique que nous délimiterons l'étude du Cycle de l'Invisible d'Eric-Emmanuel Schmitt, tout en proposant quelques pistes de réflexions.

Charles Mauron, influencé par la psychanalyse freudienne, établit les fondements de la psychocritique. Mauron souligne l'importance de détecter un fait négligé dans la vie de l'auteur, un thème central qui s'émancipe de tout acte conscient. Selon Mauron, «il faudra préciser le rôle de cet ébranlement affectif premier »? Qu'est ce que la méthode psychocritique ? Comment parvient-elle à détecter les éléments dissimulés dans l'inconscient de l'auteur? Comment peut-elle élucider la formulation de l'inconscient à travers le matériau conscient de l'auteur qui est l'œuvre?

La psychocritique est une méthode d'analyse qui étudie dans une œuvre des faits issus de la personnalité du personnage. Elle recherche les associations d'idées involontaires sous les structures voulues du texte. Cette méthode de critique littéraire s'appuie donc sur le décèlement de la structure fondamentale autour de laquelle s'organise la production littéraire de l'écrivain. En d'autres termes, il s'agit de découvrir les motivations inconscientes de l'auteur à travers son œuvre. En suivant le fil des associations d'idées et en étudiant ce réseau complexe d'images, nous parviendrons à établir un réseau, c'est-à-dire un système de relation entre les mots et les images.

${ }^{5}$ En fait, cette idée-force anime les quatre romans piliers de l'œuvre de Schmitt. ; Milarepa est consacré au bouddhisme, Monsieur Ibrahim et les fleurs du Coran se base sur l'Islam, Oscar et la dame rose s'inspire du christianisme et L'enfant de Noé traite le judaïsme. Chaque récit de ce cycle aborde un drame humain et le lie à une religion et à la sagesse spirituelle. Cf. LISOVA, Martina, L'enfant au cour du cycle de l'invisible, Faculté de Pédagogie, Université Masarykova, Tchèque, 2009.

${ }^{6}$ SCHMITT, Eric-Emmanuel, Le Visiteur, suivi de l'Interview exclusive de CASINPELLEGRINI, Catherine avec Eric-Emmanuel Schmitt, Magnard, Paris, 2002, p.134.

${ }^{7}$ MAURON, Charles, Des métaphores obsédantes au mythe personnel, Introduction à la psychocritique, Paris, Corti, 1963, p.23. 
Mauron affirme : «Le symbole exprime à la fois l'inconscient inférieur et la spiritualité supérieure $\gg{ }^{8}$

Il s'agit d'abord de commencer par la superposition de textes qui mène aux réseaux d'associations et aux groupements d'images obsédantes et involontaires. Pour y parvenir, nous proposons de superposer les différents types de personnages ; en particulier Freud et l'Inconnu. A travers l'interaction de ces deux personnages, nous parviendrons à déterminer les caractéristiques de chacun d'eux. A travers l'œuvre, le mythe personnel est dégagé par la recherche des modifications des structures des situations. La méthode propose donc « un système de langage conscient et de langage inconscient, le langage combine plusieurs logiques à la fois, comme le critique, qui passe lui-même du freudisme à la littérature ${ }^{9}$. Le mythe personnel reflète la personnalité inconsciente de l'auteur. Il renvoie à une «situation dramatique interne, sans cesse modifiée par des éléments externes, mais toujours reconnaissable et persistante $\gg$.

La dernière étape consiste à faire une correspondance avec la vie de l'écrivain et à établir un contrôle biographique. La biographie vérifie l'analyse du texte. Mauron souligne :

«Les processus inconscients d'un individu humain dépend, dans une certaine mesure, et à travers des retentissements compliqués, des événements dès son existence. Dans la mesure encore ou la vie imaginative dépend à son tour des processus inconscients, elle est fonction des événements biographiques » ${ }^{11 .}$

Schmitt ancre sa pièce dans un contexte historique. Toute la pièce se déroule en temps réel. En fait, il choisit d'évoquer les années que Freud a passées à Vienne lors de l'occupation allemande pendant la deuxième guerre mondiale plus particulièrement «le soir du 22 avril 1938, c'est-à-dire entre l'invasion de l'Autriche par les troupes hitlériennes (11 mars) et le départ de Freud pour Paris (4 juin) » ${ }^{12}$.

L'action a lieu dans le cabinet du Docteur Freud qui après l'arrestation de Anna, sa fille, se trouve seul, face à ses craintes,

${ }^{8}$ Ibid, p. 143.

9 Ibid, p. 156.

${ }^{10}$ Ibid, p. 154.

${ }^{11}$ Ibid, p. 211.

${ }^{12}$ Le Visiteur, p.14. 
jusqu'à l'irruption énigmatique de ce visiteur, de cet Inconnu, dont la présence suscite tant d'interrogations. Qui est-ce donc ce deus ex machina $^{13}$ ? Un voleur?

Freud s'adresse à l'inconnu :

«Que venez vous faire ici ? (Croyant comprendre qu'il s'agit d'un voleur) Il n'y a plus d'argent, vous arrivez trop tard ». ${ }^{14}$

Le Nazi qui vient menacer Freud, propose une autre hypothèse :

«Un cinglé. Mais il n'est pas dangereux; je crois que c'est un de ces types qui se racontent des histoires, vous savez, le genre qui se prend pour Goethe ou pour Napoléon.

Freud (Avec angoisse) : Un mythomane ! ». ${ }^{15}$

Est-ce un malade, un névrosé ${ }^{16}$, qui demande les soins d'un médecin ?

Est-ce au contraire, un visiteur qui vient assister Freud malade et prisonnier dans sa solitude ? Est-ce un bon écrivain perspicace qui a accès aux tréfonds des sentiments les plus intimes de Freud ? Qui-est cet inconnu qui connaît les événements et qui a la capacité d'accéder à la pensée des autres?

«Freud: Qui êtes-vous? Qui êtes-vous?

L'Inconnu (légèrement) : Vous ne me croiriez pas ». ${ }^{17}$

La profusion de structures interrogatives et exclamatives sans réponses, adressées à l'Inconnu dont l'identité est jusque là non identifiée, n'est que le reflet d'un Moi brisé, d'un Moi qui exprime son angoisse et cherche un refuge au sein même d'une présence angoissante.

Toutes ces hypothèses sont réfutées. L'Inconnu n'est pas un voleur et le fou a été retrouvé. Tout au long de la pièce Freud oscille entre les différentes identités qu'il essaye d'attribuer à l'Inconnu.

Freud essaye de convaincre Anna, sa fille, de la présence de cet Inconnu :

\footnotetext{
${ }^{13}$ Ying HSIEH, Yvonne, Eric-Emmanuel Schmitt ou la philosophie de l'ouverture, Suma Publications Inc., Birmingham, 2006, p.19.

${ }_{15}^{14}$ Le Visiteur, p.30.

15 Ibid., p.61.

16 La névrose est une psychopathologie caractérisée par des troubles psychiques et des symptômes corporels qui résultent du refoulement des pulsions obsessionnelles infantiles notamment sexuelles. Le sujet est conscient de sa souffrance psychique et ne présente ni délire ni hallucinations ; il vit dans la réalité

${ }^{17}$ Le Visiteur, p.30-31.
} 
«Freud : Un inconnu ! Un visiteur qui mérite d'être connu, je ne peux t'en dire plus. (Cherchant désespérément partout) Mais voyons, il n'est pas sorti... ni par la porte, ni par la fenêtre !

Nous parlions lorsque tu es rentrée.

Anna: Tu étais seul.» ${ }^{18}$ Anna essaye de convaincre son père qu'il dormait et que cette présence n'est qu'un songe ou un rêve. Cette hypothèse est aussi brutalement écartée par un coup de théâtre. Anna reconnaît l'Inconnu ; c'est l'homme qui la suit depuis quinze jours. Ce qui rend la situation critique c'est qu'Anna parvient à voir l'Inconnu qui était jusque là invisible.

Freud supplie l'Inconnu d'intervenir auprès du Nazi :

«Restez avec moi ! Parlez-lui !

L'Inconnu : Ridicule ! De toute façon, il ne peut pas me voir. Je ne suis visible que pour toi, ce soir. " ${ }^{19^{3}}$

Est-il réel ? Est-ce un spectre ? Un fantôme ? Une apparition?

En fait, la recherche de l'identité est complexe. Nous pouvons entrevoir deux sens majeurs à la question de l'identité : une face objective et une face subjective. La face objective est le fait pour une personne d'être tel individu et d'être reconnu pour tel. Tandis que la face subjective, est la conscience qu'a l'être de rester le même individu tout le long de sa vie. L'identité est à la fois personnelle et sociale. Elle exprime la singularité de l'individu et son appartenance à une catégorie (sociale, familiale, ethnique, idéologique, religieuse) qui détermine les caractéristiques identitaires de l'individu. Alain RobbeGrillet affirme :

«Un personnage doit avoir un nom propre, double si possible : nom de famille et prénom. Il doit avoir des parents, une hérédité. Il doit avoir une profession [...] Enfin, il doit posséder un caractère, un visage qui le reflète, un passé qui a modelé celui-ci et celui-là ». ${ }^{20}$

L'Inconnu ne possède ni une identité individuelle fixe ni une identité sociale déterminée. Freud perd alors tous ses repères devant cette crise identitaire. $^{21}$

\footnotetext{
${ }^{18}$ Le Visiteur, p.91.

19 Ibid., p.56.

${ }_{20}$ ROBBE-GRILLET, Pour le nouveau roman, Editions de Minuit, 1963, Paris, p.27.

${ }^{21}$ Cf., COSTALAT-FOUNEAU, Anne-Marie, LIPIANSKY, Edmond-Marc, «Éditorial. « Le sujet retrouvé »», in Connexions, $\mathrm{n}^{\circ} \quad 89, \quad$ janvier 2008, p. 7-12. www.cairn.info/revue-connexions-2008-1-page-7.htm r..
} 
Face à cette énigmaticité, Schmitt renvoie son spectateur à une situation irréductible. Qui est donc cet Inconnu dont l'apparition s'avère problématique ?

«Qui est-il ? Que va-t-il devenir désormais, maintenant que l'incertitude et l'indétermination sont devenues son destin, parabole de notre société qui a perdu elle aussi ses repères d'antan et ses certitudes ? $\gg^{22}$

En fait, tout le long de la pièce, Freud cherche en vain à trouver des justifications et lorsqu'il croit parvenir à trouver des réponses à ses questions, l'Inconnu intervient :

«L'Inconnu (Avec une moue) : Je vous préférais lorsque vous posiez des questions. $»^{23}$

Freud ne tarde pas alors à reprendre son interrogatoire. Mais ses questions ne sont que l'écho d'un discours personnel et d'une interrogation intérieure. Freud tente de trouver des réponses et des liens logiques afin d'apaiser son angoisse. Cela trouble le spectateur puisque Freud est supposé être la personne qui donne les réponses. Ce qui aggrave encore plus la problématique de la pièce. Meyer souligne ainsi l'originalité de Schmitt :

«Schmitt se révèle ainsi le plus philosophe des écrivains, mais il est en avance sur son temps. Ce que la pensée commence à entrevoir - à savoir la nécessité de penser le questionnement plutôt que celle de vouloir toujours obtenir des réponses qui prétendent l'abolir, Schmitt, lui, le développe par la fiction avec une force et une pertinence rares.» 24

Le spectateur est ainsi confronté à un mystère ; les supposées réponses se transforment, à leur tour, en questions. L'Inconnu change d'identité à chaque fois que Freud semble frôler la vérité. L'Inconnu est ainsi « le signe vivant des réponses qui s'effritent parce qu'il brise les certitudes et les valeurs les plus ancrées.» ${ }^{25}$

Selon Schmitt, les questions font avancer l'homme puisque l'existence est un questionnement et les réponses sont trompeuses, relatives, et sujet à un changement continuel.

\footnotetext{
22 MEYER, Michel, op.cit., p.22.

${ }^{23}$ Le Visiteur, p.30.

${ }^{24}$ MEYER, Michel, op.cit., p.154.

${ }^{25}$ Ibid., p. 19.
}

$$
\mu \cdot 1
$$


«Freud : Les enfants sont spontanément philosophes : ils posent des questions.

Anna : Et les adultes?

Freud : Les adultes sont spontanément idiots : ils répondent. ${ }^{26}$

L'homme vit dans un monde de réponses jusqu'au jour où il finit par voir toutes ses certitudes s'ébranler.

Schmitt projette sur scène le Moi freudien à travers le personnage de Freud. Le Moi, dans Le Visiteur, perd ses assurances, s'effrite sous le poids de l'Histoire. La primauté de la subjectivité a cédé la place aux grandes interrogations. Freud perd alors son repère le plus stable, son Moi. Son dialogue avec l'autre est une autoanalyse, un prétexte pour se comprendre.

«Freud : Vous tombez mal ce soir, il n'y a plus de docteur Freud... Guérir les autres... Croyez-vous que soigner les hommes m'empêche, moi, de souffrir? Il est même des soirs ou j'en veux presque aux autres de les avoir sauvés; je suis si seul, moi, avec ma peine, sans secours... ${ }^{27}{ }^{27}$

Freud, considéré comme un individu hors du commun, est traversé par les mêmes incertitudes ainsi que les mêmes questions que tout le monde redoute.

«Freud (pour lui-même) : Je deviens fou.

L'Inconnu : La sagesse consiste souvent à suivre sa folie.» ${ }^{28}$

Freud oscille entre les différentes identités qu'il essaye d'attribuer à l'Inconnu. Est-ce un malade, un voleur, une victime ou un simulateur? Freud est indécis jusqu'au moment où L'Inconnu commence à prédire l'avenir.

«L'Inconnu : Vous serez à Paris, chez la princesse Bonaparte... puis à Londres, à Maresfield Gardens... si ma mémoire est bonne...

Freud : Maresfield Gardens ?... mais... vous pouvez dire ce que vous voulez, je n'en sais rien... je n'ai rien prévu...

L'Inconnu : Si, si. Vous y serez bien. ${ }^{29}$

\footnotetext{
${ }^{26}$ Le Visiteur, p.17.

${ }^{27}$ Ibid., p. 35 .

${ }^{28}$ Ibid., p.43.

${ }^{29} \mathrm{Ibid}$, p.33.En fait, Maresfield Gardens à Londres fut la dernière résidence de Freud. La maison est devenue ensuite un musée. 
Les paroles de l'Inconnu commencent à semer le doute dans l'esprit de Freud. L'Inconnu a-t-il recours à sa mémoire pour visionner l'avenir?

La conversation devient encore plus curieuse lorsque l'Inconnu affirme qu'Anna, la fille de Freud, arrêtée par la Gestapo, reviendra.

«L'Inconnu : Elle reviendra (Freud a un geste interrogatif) Anna [...] Et vous la tiendrez dans vos bras, lorsqu'elle reviendra... $»^{30}$

Schmitt réintègre, à travers Freud, l'Humanité et ses personnages incarnent l'expression de nos questions les plus intimes. C'est surtout cette identité partagée qui rend le théâtre de Schmitt universel.

Le monde est problématique et toute la pièce se profile dans cette vision. Schmitt trouve là son point d'ancrage. L'Inconnu incarne le monde. Il est une métaphore de l'Histoire qui bouscule les identités, les mélange et bouleverse tout sur son passage. ${ }^{31}$ Plus l'Histoire avance, plus l'identité de l'Inconnu devient confuse. Schmitt excelle donc à transmettre au spectateur cette perception du métaphorique.

Face à cette perte totale de l'identité de l'Inconnu, et, dans une dernière tentative de formaliser et d'appréhender la problématique de l'identité de ce visiteur, le recours aux méthodes d'analyse psychanalytique s'avère indispensable. C'est à ce moment que Freud décide de reprendre son rôle de praticien et de repousser le moindre soupçon d'irrationnel et d'invisible.

Il faut là noter l'influence de la psychanalyse sur les ouvrages de Schmitt. Le dramaturge s'attache à mettre en lumière l'inconscient de ses personnages. En suivant la pièce, le lecteur a un mode d'emploi de la psychanalyse et des méthodes psychothérapiques de Sigmund Freud. ${ }^{32}$

Freud commence par appliquer sa psychothérapie sur l'Inconnu allongé sur le divan. ${ }^{33}$ Cette pratique ne tarde pas à échouer devant les réponses du patient : «Je n'ai pas de père. Personne ne m'appelle. Je ne rêve jamais. ${ }^{34}$ Devant ce que Freud appelle verrouillage de la

\footnotetext{
${ }^{30}$ Ibid., p.35.

31 Cf. MEYER, Michel, op.cit., p.12.

${ }^{32}$ Cf. POLIGONE, Pierre, La philosophie facile de Schmitt, 2014, www.zonecritique.com

${ }^{33}$ Pour que le patient associe plus librement, il est allongé sur un divan. Cette position qui évoque le sommeil favorise l'émergence de l'imaginaire.

${ }^{34}$ Le Visiteur, p.37. 
mémoire $^{35}$ par la censure, il décide de changer de technique. Lors de la session de défoulement, l'inconnu s'identifie à Freud.

Freud et l'Inconnu (l'Inconnu prononce en même temps que lui les mots sur les lèvres) : «Je suis Sigmund Freud, j'ai cinq ans, j'existe ; il faudra que je me souvienne de ce moment-là.»36

Il est intéressant de remarquer que les voix narratives sont entrelacées. Elles participent à la production du récit de l'histoire racontée, ainsi que la structure inconsciente qui les sous-tend. L'Inconnu parvient à ranimer les complexes infantiles de Freud. Nous assistons ici à une superposition de ces deux personnages. L'utilisation du présent renforce la continuité dans le temps et accentue l'ancrage de Freud dans le passé. Le fait est vrai et il dure encore au moment de la parole. Sigmund Freud a longtemps valorisé les vertus du refoulement, considérées comme une ligne de défense. ${ }^{37}$

L'Inconnu est parvenu à dépasser cette barrière. L'inquiétante étrangeté que ressent Freud est due à la pression qu'exerce l'Inconnu. Le dialogue ici n'est plus un dialogue avec un alter ego, mais plutôt un dialogue avec soi-même. Freud se rappelle son passé et vit un moment d'extrême confusion. Ce «monologue autobiographique ${ }^{38}$ agit comme une confession. Est-il possible de trouver un lien entre l'Inconnu et la personnalité de l'écrivain ? Peut-il être l'incarnation du Moi de l'auteur, surtout que cet Inconnu ne possède aucune référence sociale? En fait, nous assistons ici à une fusion entre les deux protagonistes. L'Inconnu ne serait-il pas que la voix intérieure de Schmitt ? Ce visiteur ne serait-il pas le reflet de l'âme tourmentée et de l'esprit troublé de l'auteur?

A travers l'étude de Freud et de l'Inconnu, nous remarquons la présence d'un lien entre la structure du texte et l'inconscient de l'auteur.

A ce niveau de notre travail, nous avons démontré à travers l'analyse des personnages clés et les instances du corpus qu'il existe

\footnotetext{
${ }^{35}$ Mise en œuvre du refoulement qui opère en travestissant les désirs refoulés de manière à les rendre méconnaissables.

${ }^{36}$ Le Visiteur, p.39.

${ }^{37}$ Cf. FREUD, Sigmund, Essais de psychanalyse appliquée, Gallimard, Paris, 1971

${ }^{38} \mathrm{COHN}$, Dorrit, La transparence intérieure, modes de représentation de la vie psychique dans le roman, Seuil, Paris, 1981, p.208. 
un lien entre le texte et l'inconscient de l'auteur. La dernière partie de l'analyse sera consacrée à clarifier le mythe personnel de l'auteur. Nous tenterons ensuite d'étudier les tendances refoulées du Moi, et à éclaircir les motifs de leur apparition ainsi que leur reflet sur la vie de l'auteur. Nous articulerons notre analyse sur La nuit de feu.

Nous avons tenté de démontrer à partir de l'étude des personnages et leur relation qu'il existe un lien entre le texte et l'inconscient de l'auteur. Quelle angoisse obsède-t-elle Schmitt au point de le mener à cet état ? L'ensemble des souvenirs de l'auteur ainsi que les données biographiques peuvent donner les explications nécessaires. Jean Bellemin-Noël affirme que l'analyse consiste à «décrire les obsessions ou les mythes personnels qui semblent se placer au centre d'une thématique $»{ }^{39}$

Mais au-delà des questions irréductibles qui hantent le théâtre de Schmitt, le dramaturge fait appel à l'irrationnel, à l'invisible, à l'audelà de l'évidence, à Dieu. C'est là que se noue la vision de Schmitt du Cycle de l'Invisible. Meyer explique la conception de Schmitt de l'Invisible :

«Dieu est par définition au-delà du visible et de la pensée qui ne fonctionne que par des propositions en guise de réponses. Pour lui, chaque réponse renvoie à des questions, à des questions de questions, et Dieu n'est jamais qu'un mot, un espace blanc, pour capturer cette interrogativité infinie qui caractérise l'homme.»» ${ }^{40}$

Le Cycle de l'Invisible exprime non seulement l'énigmaticité du monde mais aussi la force de ce monde et sa violence envers la faiblesse humaine. L'homme cherche à faire face aux évidences qui dépassent sa force (maladies, pertes, désespoir, mort). Il s'adresse donc aux religions afin de retrouver le Salut. Vu sa faiblesse, l'homme est obligé d'aller au-delà des réponses et de commencer sa propre quête.

Qu'est-ce donc l'Invisible ? L'Invisible est ce que l'on ne voit pas et qui nous fait agir. C'est tout ce qui transcende le visible. C'est tout ce qui incite la pensée à ne pas se limiter à ce qui se présente

\footnotetext{
${ }^{39}$ BELLEMIN-NOEL, Jean, Vers l'inconscient du texte, Paris, Presses universitaires de France, Paris, 1979, p.11.

40 Ibid., p.49.$$
\text { r.o }
$$ 
directement au regard de l'homme. C'est cet excédent d'interrogations qui se posent face aux réponses les plus sûres. C'est cet appel à Dieu. Faut-il appeler la source «Dieu»? «Oui, si c'est le nom d'une distance incompressible. Dieu est alors le nom de l'inconnu, de la question pure qui rebondit sans cesse au détour des posivitivités de la vie quotidienne, comme un manque inscrit au cœur du réel, comme le signe d'une réalité qui s'y cache ». 41

Il faut noter que Schmitt est devenu croyant le 4 Février 1989, lors d'un voyage dans le désert du Hoggar au Sahara.42 Perdu, seul dans le désert, sans rien à boire ni à manger, il a trouvé la foi. Cette nuit au lieu de lui causer peur et angoisse, a été au contraire, une nuit qui lui a donné une confiance absolue dans la vie et dans le mystère de la condition humaine. Schmitt affirme :

«J'ai été incendié par la foi, irradié, pénétré par une force tellement plus grande que moi que je ne pouvais pas en être l'auteur.» ${ }^{43}$

Schmitt précise qu'il s'est détaché de l'absurdisme. Il renoue aussi avec l'idée que ce qui lui échappe n'est pas nécessairement du non-sens. La rationalité était incapable de répondre à toutes ses questions. ${ }^{44}$ Schmitt ajoute :

«J'ai éprouvé le sentiment de l'Absolu et, avec la certitude qu'un ordre, une intelligence, veille sur nous, et que, dans cet ordre, j'ai été créé, voulu. Et puis la même phrase occupait mes pensées. Tout est justifié.» 45

Mais, pourquoi Schmitt a-t-il choisi Freud comme héros à sa pièce; Freud, cet athée radical, aux convictions inébranlables, «catéchumène de l'incroyance» ${ }^{46}$ comme le qualifie l'Inconnu ?

Il est de même convenu que le freudisme supprime la transcendance chez 1'homme. A travers sa psychanalyse, Freud cherche à découvrir

${ }^{41}$ Ibid., p. 108

${ }^{42}$ Cf. Eric-Emmanuel Schmitt, La nuit de feu, Albin Michel, Paris, 2015.

${ }^{43}$ QUERRY, Emmanuel, «Entretien avec Eric-Emmanuel Schmitt » in L'Invisible, n. 42 , Novembre 2013, p.7

${ }^{44}$ Cf. ALBIN, Michel, Eric-Emmanuel Schmitt, le philosophe en parabole in L'Homme en question, n.23, 2009, p.2.

${ }^{45}$ LESEGRETAIN, A., «Entretien avec Eric-Emmanuel Schmitt, Ce que j'écris me dépasse » in La Croix, 2000. Version électronique : www.eric-emmanuelschmitt.com / Portrait presse.html

${ }^{46}$ Le Visiteur, p.44.

$$
\Gamma \cdot T
$$


les pulsions de l'être humain, d'agencer le champ pulsionnel afin de parvenir à trouver une réponse, une justification rationnel et explicable à toutes sortes de mystères impénétrables. Il est donc impératif pour Freud de rendre tout à l'intellect. De plus, c'est l'époque ou Freud vient d'achever son ouvrage Moïse et le Monothéisme où il cherche à prouver que la religion que donna Moïse aux Juifs est celle d'Ikhnaton; la religion d'Aton. Il considère le Christ comme son successeur et une réincarnation de l'image du Père primordiale. Dans cet ouvrage Freud démontre l'analogie qui existe entre le processus névrotique et les faits religieux. Il transpose la psychologie individuelle en psychologie collective. ${ }^{47}$ Dans Le Visiteur, Schmitt nous montre le parcours que fait Freud et les étapes par lesquelles il passe lors de son entretien avec l'Inconnu au cours de la soirée du 22 avril 1938. Quoi de plus intéressant donc que de choisir Freud pour défendre son athéisme et soulever une des plus grandes querelles devant son antagoniste : l'Inconnu ?

Retourner au passé nous permet d'analyser les ressemblances entre certains éléments de la vie de l'auteur et le réseau d'association d'idées présent dans le corpus étudié. En d'autres termes, les souvenirs marquants dans la vie de l'auteur influencent inconsciemment la création littéraire. Il est indispensable donc d'étudier la vie sociale et familiale de l'auteur. Cependant il ne s'agit pas de tracer la biographie de l'auteur ou d'établir son portrait. Les analyses seront focalisées surtout sur les souvenirs susceptibles d'avoir formé la personnalité de l'auteur. Il est important de détecter le déclencheur de l'événement. Freud le désigne par la notion de «l'après coup ${ }^{48}$. Cette notion fait référence aux névroses déclenchées dans la vie adulte mais, dont le traumatisme prend source dans l'enfance et il est amené inconsciemment à la surface. Cet après coup nous le détectons dans La nuit de feu lorsque Schmitt découvre la présence de Dieu :

«Dieu, je l'ai atteint par le cœur. Ou Il a atteint mon cœur. Là, en moi, s'est creusé un corridor entre deux mondes, le notre et le Sien. J'ai la clé, le chemin. Nous ne nous quitterons plus. Quel bonheur

\footnotetext{
${ }^{47}$ Freud, Sigmund, Moise et le monothéisme, Québec, 2002, version électronique : www.uqac.uquebec.ca/zone30/classiques_des_sciences_sociales/index.html

${ }^{48}$ FREUD, Sigmund, L'hérédité et l'étiologie des névroses in OCEP, 1896, p.118. $r \cdot v$
} 
qu'il existe ! Joie ! Par ma foi toute neuve, je l'éprouve d'une façon puissante. $\gg{ }^{49}$

Schmitt commence, ici, à introduire la notion de foi, de Dieu et du Cycle de l'Invisible. Le sentiment d'abandon que Freud ressent, alors qu'il n'avait que cinq ans dans cette vaste maison, est à l'origine de son athéisme puisque personne n'est venu le secourir lorsqu'il a appelé. Face au sentiment d'abandon et de solitude éprouvée par Freud-enfant, il s'écrie :

«Et le monde est cette vaste maison vide où personne ne répond lorsqu'on appelle.») 50

Schmitt enfant est marqué par l'athéisme de ses parents et cela peut être considéré comme une clef pour la reconstruction de son mythe personnel puisque les traumatismes psychologiques de l'enfance ont formé sa personnalité. Schmitt affirme :

«J'ai grandi dans un milieu athée et n'ai effectivement reçu aucune éducation religieuse. ${ }^{51}$

Schmitt reprend dans La nuit de feu le dialogue qu'il a entretenu avec son père :

«- Où est Dieu dans tout ça ?

Le visage de mon père se contracta. Une sorte de vide envahit ses prunelles. Il semblait déçu, dégouté. Il se gratta le crâne et finit par confesser d'une voix lasse :

- Dieu n'est nulle part. Moi, je ne le vois pas. ${ }^{52}$

En tant qu'adulte, Schmitt garde la même attitude envers la présence divine. Le monde est vaste et Dieu est absent :

«Je regardai le désert sombre autour de moi.

Où est Dieu dans tout ça ?

Moi non plus, je ne le voyais pas... » ${ }^{53}$

\footnotetext{
${ }^{49}$ La nuit de feu, p139.

50 Le Visiteur, p.39.

51 NOYE, Jean-Claude, Entretien avec Eric-Emmanuel Schmitt: "Le mystère ou Dieu se tapit » in Prier, publié le 1/12/2009.

www.prier.presse.fr/dossiers/people/eric-emmanuel-schmitt-le-mystère-ou-dieu-setapit-01-12-2009-666-141.php

${ }^{52}$ La nuit de feu, p.75.

53 Ibid., p.77. 
La technique de 1 'hypnose ${ }^{54}$ pratiquée par Freud ne vaut pas mieux puisque les réponses de l'Inconnu continuent à être de plus en plus mystérieuses. La situation s'aggrave lorsque qu'il prévient Freud de la date de sa mort. Et, Freud, dans une dernière tentative de refus et de reniement, dit :

«Freud (le regard toujours dans la glace, un temps, contemplant l'inconnu) : Vous m'excuserez : je ne peux pas croire que c'est vous.

L'Inconnu : Je le sais. Tu ne crois pas en moi. Le docteur Freud est un athée, un athée magnifique...» ${ }^{55}$

L'Inconnu commence ensuite à entreprendre un langage freudien. Malgré les divergences entre les deux personnages, il est à noter que les deux personnages éprouvent le même sentiment, les mêmes souffrances. A tour de rôle, ils se dédoublent ou s'effacent l'un derrière l'autre. Le réseau structurel est le même pour les deux personnages. Ce qui nous permet d'affirmer qu'il relève d'un fantasme inconscient qui ranime toute l'œuvre.

Il explique à Freud la théorie de l'invention d'un dieu-père ${ }^{56}$ et Freud ne nie pas :

«C'est cela ${ }^{57}$ répond t-il aux justifications de l'Inconnu.

Par contre, quand l'Inconnu commence à raconter à Freud l'état où se trouve Anna qui risque d'être déportée avec les autres juifs ou fusillée, Freud se trouve dans un état qui dépasse son entendement. Il s'adresse à l'Inconnu comme un croyant qui implore le secours de Dieu lors d'une crise.

«Freud a un cri de bête et se précipite sur 1'Inconnu, l'attrapant par le col

Freud : Faites quelque chose ! Intervenez vite $!{ }^{58}$

\footnotetext{
${ }^{54}$ Technique utilisée par Freud dans le traitement des patients mais qui fut abandonné au profit de la technique de l'association libre. Durant la séance, la parole appartient au patient. Il doit dire ce qui lui passe par l'esprit, sans trier les pensés et les images. C'est la règle fondamentale de la psychanalyse freudienne.

${ }_{56}^{55}$ Le Visiteur, p.44.

${ }^{56}$ Selon Sigmund Freud, l'homme, frustré par l'absence d'un père idéal, le remplace par l'entité divine. Afin de compenser le sentiment de culpabilité ressenti après le meurtre du père, l'homme invente la conception du dieu-père. Cf. Moise et le Monothéisme, op.cit.

${ }^{57}$ Le Visiteur, p.48.

58 Ibid., p.53. 
Lors de l'apparition du Nazi, et face à sa menace de perdre tout son argent, Freud demande une seconde fois l'assistance de Dieu. Il se raccroche alors à Dieu afin de parvenir à faire face au danger. Freud est dans un état d'affaiblissement du Moi.

Devant l'éventualité de perdre sa fille ou sa fortune, Freud abandonne ses théories athéistes et se tourne instinctivement vers Dieu.

Freud semble sur le point d'admettre l'existence de Dieu mais Schmitt choisit de construire sa pièce autour du doute et la présence de plusieurs mots de négation traduit cette remise en question.

«Freud (violent) : Je ne crois pas en Dieu parce que tout en moi est disposé à croire! Je ne crois pas en Dieu parce que je voudrais y croire ! Je ne crois pas en Dieu parce que je serais trop heureux d'y croire ! » 59

Freud va même jusqu'à accuser Dieu de promesses non-tenues. Dans ses commentaires personnels, Schmitt exprime ce doute, qu'il avait lui aussi, ressenti un jour :

«J'avais écrit ce texte dans une grande solitude, selon une nécessité intérieure [...] Comment croire en Dieu aujourd'hui ? [...] Comment croire en Dieu à l'insu de ce XXe siècle si meurtrier ? Comment croire en Dieu face au Mal? $»^{60}$

Et quoi de plus problématique que ce sentiment de doute qui envahit l'homme ? Freud s'écrie devant le Mal qui ravage le monde :

«Freud : Et vous les laissez les faire!

L'Inconnu : J'ai fait l'homme libre.

Freud : Libre pour le mal !

L'Inconnu: Libre pour le bien comme pour le mal, sinon la liberté n'est rien. » ${ }^{61}$

Schmitt reprend ici la question de Leibniz dans ses célèbres Essais de Théodicée où il s'aventure à plaider la cause de Dieu. Cette doctrine a pour but de disculper Dieu du mal qui se rencontre dans le monde. ${ }^{62}$ L'Inconnu se défend ou défend Dieu :

\footnotetext{
${ }^{59}$ Ibid., p.66.

${ }^{60}$ Cf. www.eric-emmanuel-schmitt.com / Portrait presse.html

${ }^{61}$ Le Visiteur, p.85.

${ }^{62}$ La Théodicée de Leibniz repose sur l'idée que se qui semble être un mal est en réalité un bien. Il suffit de le considérer d'une autre perspective. Le mal est donc un r.
} 
«Si tu pouvais voir, comme moi, à l'avance, le ruban des années à venir, tu serais plus virulent encore mais détournerais ton accusation vers le vrai responsable. Ce siècle sera l'un des plus étranges que la terre ait portés. On l'appellera le siècle de l'homme, mais ce sera le siècle de toutes les pestes... ${ }^{63}$

Schmitt ouvre ce débat entre Freud et le mystérieux visiteur dans un climat difficile, ce qui ajoute une ampleur encore plus dramatique à ce duel. ${ }^{64}$ La responsabilité et la liberté de l'homme par rapport au choix du Mal et du Bien est mise en question. Les deux protagonistes s'accusent. Freud se déchaîne contre le silence de Dieu devant l'atrocité du monde et va jusqu'à le supplier d'intervenir. De l'autre côté, Dieu va plaider contre le libre-arbitre de l'homme qui est le seul responsable de ses actes.

Tout au long de la pièce, Schmitt sème le doute. Les personnages se dérobent à la certitude.

L'Inconnu après avoir presque convaincu Freud de la présence de Dieu, ébranle cette certitude :

«L'Inconnu (Sur le ton de la confidence) : Dites-moi, tout à l'heure, vous avez réellement cru que j'étais... (Montrant le ciel)... Lui ?

Freud(Honteux) : J'ai perdu pied ${ }^{65}$

En effet, la récurrence de ce doute démontre l'impact qu'a ce souvenir dans le psychisme de Schmitt. Il ne peut s'empêcher de l'extérioriser. Cet événement est en réalité la source qui tisse la trame suivie inconsciemment par l'auteur, d'où la naissance du mythe personnel de Schmitt. Quelles sont les caractéristiques de ce mythe ?

moyen pour atteindre un grand bien. Dieu a créé le meilleur des mondes possible.

Cf. http://gallica.bnf.fr/ark:/12148/bpt6k6526466z

${ }^{63}$ Le Visiteur, p.73.

Le $\mathrm{XX}^{\mathrm{e}}$ siècle fut le plus meurtrier de l'Histoire. Le bilan humain des massacres et des génocides est dramatique. Cf. http://www.le-cartographe.net/dossierscarto/monde/34-mon-travail/monde/104-les-pertes-militaires-des-guerres-du-xxemesiecle

file:///C:/Users/hp-/Downloads/un-siecle-de-guerres-de-massacres-et-degenocides.pdf

${ }^{64}$ Le fond historique de la pièce est l'invasion de l'Autriche par l'Allemagne lors de la deuxième guerre mondiale par les troupes nazistes ainsi que le début de

l'holocauste. Cf. https://www.herodote.net/12_mars_1938-evenement-

19380312.php

${ }^{65}$ Le Visiteur, p.64. 
A quel moment intervient-il dans le processus de la création littéraire ? Contribue-t-il à la naissance et au développement du «Moi créateur $\gg 66$ de l'écrivain ? Selon Mauron, la recherche des solutions chez l'homme normal est canalisée vers les relations humaines, ce qui absorbe toute l'énergie de l'individu. Chez l'artiste, au contraire, cette énergie s'oriente vers la création littéraire. Mauron souligne :

«Assez rapidement, la bifurcation s'accentue, et le moi créateur se développe ${ }^{67}$

Cependant la réponse divine se manifeste. Schmitt l'assure puisque l'Inconnu répond :

«Je suis venu te dire que c'est faux. Il y a toujours quelqu'un qui t'entend. Et qui vient. ${ }^{68}$

Ainsi, l'écriture de Schmitt structure le mythe qui se dégage du fait qu'il a vécu dans La nuit de feu. Il affirme :

«Une certitude brille au dessus de tout : Il existe.

Qui ?

Je ne sais pas le nommer. Lui ne s'est jamais nommé.

Il existe. ${ }^{69}$

De même, dans Le Visiteur, Schmitt cherche à prouver la présence de Dieu. Puisque Dieu suscite la réflexion, l'interrogation et l'éthique de l'homme, donc il existe. Schmitt affirme :

«Ni présent, ni absent; Dieu est caché. Il nous laisse libre de croire ou de ne pas croire en lui, il suscite notre liberté. ${ }^{70}$

A la fin de la pièce, le coup de révolver raté, en ciblant l'Inconnu symbolise la rationalité qui ne parviendra jamais à épuiser le mystère. L'Inconnu affirme à Freud :

« Je suis un mystère Freud pas une énigme ${ }^{71}$

En fait, une énigme se résout, un mystère se vit. ${ }^{72}$

Le Visiteur trace non seulement les conflits personnels de l'auteur mais reflète aussi l'angoisse de l'humanité. Freud partage sa

\footnotetext{
${ }^{66}$ MAURON, Charles, Op.cit, p.227.

${ }^{67}$ Ibid, p.228.

${ }^{68}$ Le Visiteur, p.39.

${ }_{69}$ La nuit de feu, p.138.

${ }^{70}$ Le Visiteur, p.134.

71 Ibid., p.101.

${ }^{72}$ MEYER, Michel, op.cit., p.48.
} 
vision des événements historiques. Il se veut la mémoire de l'humanité. C'est là que l'inconscient de l'auteur se rejoint à sa personnalité consciente. Son malaise personnel se double d'un malaise social. Son trouble se rejoint à celui de l'univers et à celui de tous les humains.

A travers le Cycle de l'Invisible, Schmitt prépare le terrain de la croyance mais il s'arrête au seuil. Selon Schmitt, la foi est libératrice. Il la considère comme la voie de la délivrance. Il incite son lecteur à réfléchir mais il ne cherche point à le convaincre. Le lecteur est ainsi libre de franchir le seuil de la croyance ou de s'abstenir.

«Si je faisais autre chose qu'indiquer le seuil, Le Visiteur cesserait d'être une pièce philosophique et deviendrait une pièce à thèse. ${ }^{73}$

Le doute est sa méthode et la foi est sa voie : là réside toute l'originalité d'Eric-Emmanuel Schmitt.

\section{Conclusion :}

Schmitt a toujours été tiraillé par les deux côtés de son esprit : le philosophe et l'écrivain. Il a été pendant des années inspiré par la philosophie. Face aux crises et aux angoisses, elle lui traçait le chemin de la réflexion et de l'argumentation. Schmitt est parvenu à extérioriser sa souffrance. Cependant, face aux suscitations irrationnelles, la philosophie lui semble inopérante. ${ }^{74}$ Pourtant, Schmitt est parvenu, finalement, à concilier les deux dimensions. Il affirme :

«Ma devise ? Ecrivain d'abord, et philosophe ensuite. Ecrivain par vocation, philosophe par nécessité... ${ }^{75}$

L'œuvre de Schmitt passe pour une tentative de conquête de soi, ainsi qu'une aspiration aux changements sociaux. L'analyse consistait à trouver le thème central qui émane de l'œuvre en élucidant les éléments inconscients susceptibles d'avoir donné naissance à l'œuvre. Notre but était de déchiffrer la formulation de l'inconscient à partir du

\footnotetext{
${ }^{73}$ Notes personnelles d'Eric Emmanuel Schmitt : www.eric-emmanuel-schmitt.com

${ }^{74}$ Pourtant, la philosophie de Descartes l'a rendu agnostique et Schmitt a commencé ainsi à travailler son mysticisme.

Cf. DE CORTANZE, Gérard, Réception d'Eric-Emmanuel Schmitt, Séance publique du 25 mai 2013, Académie royale de langue et de littérature française de Belgique, Bruxelles, 2013, version électronique : www.arllfb.be

${ }^{75}$ Le Visiteur, p.138.
} 
matériau conscient qui est le texte. Le choix de la psychocritique de Charles Mauron comme approche pour l'analyse du Visiteur nous a semblé la plus adéquate à trouver dans le texte la personnalité inconsciente de l'auteur. Elle nous a permis de dévoiler le vrai Moi et la vrai identité de Schmitt.

Dans un premier temps, nous avons tenté de superposer les textes et d'analyser les personnages et de souligner les caractéristiques majeures de leur relation ainsi que leurs crises identitaires. A travers la confrontation des protagonistes, nous avons démontré qu'ils incarnent le Moi de l'auteur. Nous avons focalisé notre étude sur les événements susceptibles d'avoir formé la personnalité inconsciente de l'auteur. Le mythe personnel de l'auteur est ainsi dégagé. En suivant son évolution nous sommes parvenus à révéler le drame originel de l'auteur. Nous avons tenté donc dans cette recherche de ressortir le fantasme inconscient de l'auteur et les traumatismes psychiques qui sont à l'origine de sa vocation littéraire. Ces deux dernières étapes nous ont permis d'établir un lien entre les événements marquants de la vie de l'auteur et sa création littéraire. La biographie de l'auteur, les interviews personnelles, ainsi que le roman autobiographique La nuit de feu, nous ont permis de vérifier ce qui a été analysé.

En effet, Le Visiteur reflète cette vision. La pièce est marquée et même orientée par les soubresauts de sa propre vie. Le dramaturge semble tracer, à travers ses personnages, son propre cheminement dans la voie de la croyance.

$\mathrm{Au}$ terme de cette étude sur la conception de Schmitt du Cycle de l'Invisible, nous croyons pouvoir conclure que Le Visiteur ne propose aucune réponse définitive. Schmitt invite son spectateur à adopter comme lui une attitude interrogative. Il lui ouvre grandes les portes de la réflexion.

Toutes les sectes sont représentées76, tous les arguments sont exposés, toutes les voix se font entendre. Aucun personnage ne parvient à convaincre l'autre.

«Freud : Je ne suis pas converti

\footnotetext{
${ }^{76}$ Schmitt note à propos du Visiteur : « Les Juifs y voyaient une superbe méditation hassidique, les Chrétiens une fantaisie pascalienne et les athées y retrouveraient le cri de leur douleur $»$ : www.eric-emmanuel-schmitt.com

ए। $\varepsilon$
} 
L'Inconnu : Mais toi seul peux te convertir : tu es libre !»77

Schmitt accorde à ses personnages toute la liberté de s'exprimer. Aucune censure n'est exigée, aucune conviction n'est imposée. L'Inconnu affirme :

«Personne ne me voit, chacun projette sur moi l'image qui lui convient, ou qui l'obsède. $\gg 78$

Malgré le fond historique sinistre de la pièce et la situation insoutenable des personnages, Le Visiteur est loin d'être pessimiste. Schmitt puise son optimisme du fond même du malheur. Au milieu d'un monde saccagé par la violence, l'atrocité et la souffrance, l'espérance est le seul moyen d'apaiser la douleur.79 L'espérance, c'est croire en cette force majeure qui gère le monde. L'espérance réside dans cette certitude que Dieu est toujours là. C'est surtout, cette touche d'optimisme et cette quête du Bien au milieu du Mal, qui ont permis à Eric-Emmanuel Schmitt, de séduire des milliers de lecteurs à travers le monde.

Avec un style, à la fois, précis et étincelant d'originalité, Schmitt, en allant au cœur du Moi, parvient à briser les barrières imposées par les religions, les dogmes et les différences. Rien ne saurait ébrécher son amour pour l'Autre. C'est là que réside la base de son humanisme. La force de son récit ne s'inscrit-elle pas au cœur de sa quête de l'humain universel ? Ne prépare-t-il pas ainsi son lecteur à son nouveau Cycle celui de l'Humain et de l'Inhumain ?

\footnotetext{
Le Visiteur, p.111.

78 Ibid., p.98.

79 Cf., MAKHLOUF, Georgia, Eric-Emmanuel Schmitt: « L'optimisme est la seule façon d'apaiser la douleur » in L'Orient littéraire, n.118, avril 2016. 


\section{Bibliographie :}

\section{Cuvres d'Eric-Emmanuel Schmitt :80}

1. Le carnaval des animaux, Albin Michel, Paris, 2014.

2. La chair et l'Invisible, Passiflore Editions, Paris, 2016.

3. Concerto à la mémoire d'un ange, Albin Michel, Paris, 2010.

4. Le cycle de l'invisible, coffret 5 volumes, Albin Michel, Paris, 2009.

5. Diderot ou la philosophie de la séduction, Albin Michel, Paris, 2013.

6. Les dix enfants que madame Ming n'a jamais eus, Livre de poche, Hachette, Paris, 2015.

7. L'élixir d'amour, Livre de poche, Hachette, Paris, 2016.

8. L'empoisonneuse, Albin Michel, Paris, 2016.

9. L'enfant de Noé, Magnard, Paris, 2010.

10. L'énigme Stefan Zweig, Le passeur, Paris, 2016.

11. L'évangile selon Pilate, Livre de poche, Hachette, Paris, 2006.

12. Eric-Emmanuel Schmitt, Coffret 3 volumes, Livre de poche, Paris, 2005.

13. Espaces littéraires à la recherche du bonheur, Comelsen, Paris, 2008.

14. Etranges et familiers, Avant propos, Paris, 2006.

15. La femme au miroir, Livre de poche, Hachette, Paris, 2013.

16. Frederick ou le boulevard du crime, Albin Michel, Paris, 1998.

17. Golden Joe, Albin Michel, Paris, 1995.

18. Guignol aux pieds des Alpes, Livre de poche, Hachette, Paris, 2002.

19. Guitrys, Albin Michel, Paris, 2013.

20. Histoire de ceux qui oublient ou pas, Flammarion, Paris, 2015.

21. L'homme qui voyait à travers les visages, Albin Michel, Paris, 2016.

22. Le joueur d'échecs, Magnard, Paris, 2016.

23. Le journal d'Anne Frank, Livre de poche, Hachette, Paris, 2013.

24. Le Libertin, Albin Michel, Paris, 1997.

25. Lorsque j'étais une œuvre d'art, Livre de poche, Hachette, Paris, 2004.

26. Ma vie avec Mozart, Albin Michel, Paris, 2005.

27. Ménage à trois, Albin Michel, Paris, 2016.

28. Mes évangiles, Albin Michel, Paris, 2004.

29. Monsieur Ibrahim et les fleurs du coran, Livre de poche, Hachette, Paris, 2012.

30. La nuit de feu, Albin Michel, Paris, 2015.

31. La nuit des oliviers, Compagnie des artistes, Paris, 2006.

32. La nuit de Valognes, Magnard, Paris, 2004.

33. Oscar et la dame rose, Magnard, Paris, 2006.

34. La part de l'autre, Livre de poche, Hachette, Paris, 2003.

35. Les perroquets de la place d'Arezzo, Livre de poche, Hachette, Paris, 2015.

36. Le poisson d'amour, Livre de poche, Hachette, Paris, 2016,

37. La secte des égoistes, Livre de poche, Hachette, Paris, 1996.

${ }^{80}$ Les œuvres de SCHMITT sont classées par ordre alphabétique. 
38. Si on recommencerait, Livre de poche, Hachette, Paris, 2014.

39. Sophocle, Renaissance du livre, Paris, 2004.

40. Le sumo qui ne pouvait pas grossir, Livre de poche, Hachette, Paris, 2014.

41. Ulysse from Bagdad, Livre de poche, Hachette, Paris, 2010.

42. Vingt quatre heures de la vie d'une femme, Magnard, Paris, 2016.

43. Le Visiteur, Magnard, Paris, 2002.

44. 13 récits d'enfance et d'adolescence, Magnard, Paris, 2015.

45. Etudes :

46. ALBIN, Michel, «Eric-Emmanuel Schmitt, le philosophe en parabole » in L'Homme en question, n.23, 2009.

47. BELLEMIN-NOEL, Jean, Vers l'inconscient du texte, Paris, Presses universitaires de France, Paris, 1979.

48. BESSIÈRE, Jean «L'Énigmaticité de Henry James, en passant par la réception française de son œuvre », E-rea [En ligne], 3.2 |2005, document 7, mis en ligne le 15 octobre 2005, consulté le 05 avril 2017. URL : http://erea.revues.org/554 ; DOI : 10.4000/erea.554

49. CASIN-PELLEGRINI, Catherine, «Interview exclusive » in Le Visiteur d'Eric-Emmanuel Schmitt, Magnard, Paris, 2002.

50. COHN, Dorrit, La transparence intérieure, modes de représentation de la vie psychique dans le roman, Seuil, Paris, 1981.

51. COSTALAT-FOUNEAU, Anne-Marie, LIPIANSKY, Edmond-Marc, «Éditorial. «Le sujet retrouvé »», in Connexions, $\mathrm{n}^{\circ}$ 89, janvier 2008. www.cairn.info/revue-connexions-2008-1-page-7.htm

52. DE CORTANZE, Gérard, Réception d'Eric-Emmanuel Schmitt, Séance publique du 25 mai 2013, Académie royale de langue et de littérature française de Belgique, Bruxelles, 2013, version électronique : www.arllfb.be

53. FREUD, Sigmund, Essais de psychanalyse appliquée, Gallimard, Paris, 1971.

54. FREUD, Sigmund, L'hérédité et l'étiologie des névroses in OCEP, 1896

55. FREUD, Sigmund, Moise et le monothéisme, Québec, 2002, version électronique :www.uqac.uquebec.ca/zone30/classiques_des_sciences_socia les/index.html

56. LEIBNIZ, La Théodicée,

a. http://gallica.bnf.fr/ark:/12148/bpt6k6526466z

57. LESEGRETAIN, A., «Entretien avec Eric-Emmanuel Schmitt, Ce que j’écris me dépasse » in La Croix, 2000. Version électronique :www.ericemmanuel-schmitt.com / Portrait presse.html

58. LISOVA, Martina, L'enfant au cœur du Cycle de l'Invisible, Faculté de Pédagogie, Université Masarykova, Tchèque, 2009.

59. MAKHLOUF, Georgia, «Eric-Emmanuel Schmitt: L'optimisme est la seule façon d'apaiser la douleur » in L'Orient littéraire, n.118, avril 2016. 
60. MAURON, Charles, Des métaphores obsédantes au mythe personnel, Introduction à la psychocritique, Paris, Corti, 1963.

61. MICHEL, Meyer, Eric-Emmanuel Schmitt ou les identités bouleversées, Albin Michel, Paris, 2004.

62. NOYE, Jean-Claude, Entretien avec Eric-Emmanuel Schmitt: "Le mystère ou Dieu se tapit » in Prier, publié le 1/12/2009.

www.prier.presse.fr/dossiers/people/eric-emmanuel-schmitt-le-mystère-oudieu-se-tapit-01-12-2009-666-141.php

63. POLIGONE, Pierre, La philosophie facile de Schmitt, 2014, Www.zonecritique.com

64. QUERRY, Emmanuel, «Entretien avec Eric-Emmanuel Schmitt» in L'Invisible, n.42, Novembre 2013.

65. VILAS, Jean, «La malédiction du succès » in Les cahiers de la maison, n.103, Novembre 2007.

66. Ying HSIEH, Yvonne, Eric-Emmanuel Schmitt ou la philosophie de l'ouverture, Suma Publications Inc., Birmingham, 2006.

Sites internet :

- www.albin-michel.fr

- $\quad$ www.bnf.fr/fr/acc/x.accueil.html

- www.eric-emmanuel-schmitt.com

- www.freud.org.uk/index.html

- $\quad$ www.gallica.bnf.fr

- $\quad$ www.fr.wikipedia.org/wiki

- www.worldcat.org/ 